\title{
A semi-empirical topographic correction model for multi-source satellite images
}

\author{
Sa Xiao ${ }^{1}$, XinPeng $\operatorname{Tian}^{1}$, QiangLiu ${ }^{1,2} *$ JianGuang Wen ${ }^{2,3}$,YuShuang $\mathrm{Ma}^{1}$ ZhenWei Song ${ }^{1}$ \\ ${ }^{1}$ College of Global Change and Earth System Science, Beijing Normal University, China-(xiaosabnu@gmail.com) \\ ${ }^{2}$ State Key Laboratory of Remote Sensing Science, Jointly Sponsored by Beijing Normal University and Institute of Remote Sensing \\ and Digital Earth of Chinese Academy of Sciences, Beijing 100875, China \\ ${ }^{3}$ Institute of Remote Sensing and Digital Earth of Chinese Academy of Sciences, Beijing 100101, China
}

Commission ICWG III/IVb

KEY WORDS: Remote sensing, Topographic correction, Mountain areas, DEM, Multi-source data, 6S

\begin{abstract}
:
Topographic correction of surface reflectance in rugged terrain areas is the prerequisite for the quantitative application of remote sensing in mountainous areas. Physics-based radiative transfer model can be applied to correct the topographic effect and accurately retrieve the reflectance of the slope surface from high quality satellite image such as Landsat8 OLI. However, as more and more images data available from various of sensors, sometimes we can not get the accurate sensor calibration parameters and atmosphere conditions which are needed in the physics-based topographic correction model. This paper proposed a semi-empirical atmosphere and topographic correction model for multi-source satellite images without accurate calibration parameters. Based on this model we can get the topographic corrected surface reflectance from DN data, and we tested and verified this model with image data from Chinese satellite HJ and GF. The result shows that the correlation factor was reduced almost $85 \%$ for near infrared bands and the classification overall accuracy of classification increased $14 \%$ after correction for HJ. The reflectance difference of slope face the sun and face away the sun have reduced after correction
\end{abstract}

\section{INTRODUCTIONS}

In rugged terrain areas differences in terrain orientation often create variation in signal values between pixels with similar land cover and biophysical-structural properties as a result of differences in irradiance owing to the angle of incident illumination, and differences in radiance according to the angle of existence(Sandmeier \& Itten, 1997; Soenen, Peddle, \& Coburn, 2005). The early study shows that with the increasing of sun zenith, the domain-averaged diffuse flux proportion increases to nearly $40 \%$ (Chen, Hall, \& Liou, 2006). Because the same land cover type exists difference irradiance at the satellite, it may be cause severe classification error in the mountainous land cover. So it is prerequisite to reduce the topographic effect in mountainous areas. for further application.

The model to correct mountainous land surface reflectance can be divided into several classes: ratio model, empirical model and physics-based model. The form of ratio model is simply, but it loses a large part of information, so it can not be applied for the quantitative application. The empirical model includes cosine model, C, model SCS model(Gu \& Gillespie, 1998), and SCS+C model (Soenen et al., 2005). The physical model is based on radiative transfer equation, and considering the atmospheric conditions, BRDF, the parameters of physics model have precise meaning, but some parameters is hard to obtain, and the form is too complicated. So it applies only to the situation of well calibrated data and accurate atmosphere parameters. This paper proposed a semi-empirical model to do topographic correction for multi-source image in mountain areas and test the model with Chinese satellite, GF and HJ.

\section{METHOD AND DATA}

\subsection{Method}

The total irradiance received by the horizontal surface $E_{\text {total }}$ with Lambertian assumption can be divided into two parts: direct component $E_{d i r}$ and diffuse component $E_{\text {dif }}$ :

$$
E_{\text {total }}=E_{\text {dir }}+E_{\text {dif }}
$$

In inclined surface, the direct component and diffuse component of irradiance are affected by terrain factor such as slope and aspect, so they are different form the horizontal surface. According to Stefan and Klaus(Sandmeier \& Itten, 1997), the relationship between direct component of horizontal surface $E_{\text {dir }}$ and inclined surface $E_{\text {dir }}^{i}$ can be described by the following formula:

$$
E_{\text {dir }}^{i}=E_{\text {dir }} \frac{\cos (i)}{\cos (s z)}
$$

Where $i$ is the relative angle between sun and mountain surface, $s z$ is the sun azimuth. When $i$ is equal to $s z$, it means horizontal surface. In the meanwhile, the relationship between diffuse component of horizontal surface $E_{\text {dif }}$ and inclined surface $E_{\text {dif }}^{i}$ can be described by the following formula:

$$
E_{\text {dif }}^{i}=E_{d i f} V_{d}
$$

In processing the irradiance from sky to the land, there is another important parameter: anisotropy index. The diffuse component can be separated in two parts: the anisotropy part and isotropic part. The anisotropy component of diffuse irradiance can be modelled for topography in the same way as the direct irradiance though it is part of the diffuse irradiance.

In mountain areas, besides the direct and the diffuse component, the terrain irradiance also must be considered, especially in the valley. The neighbour irradiance is determined by the following factors: the total irradiance reaches the adjacent slope; the portion part seen from the a surface $V_{t}$; and the average reflectance of the adjacent slope. Since the total solar radiation received by the slope surface is equal to the sum of the direct radiation components and the diffuse radiation components, the 
total irradiance in the inclined surface is:

$$
E_{\text {total }}^{i}=E_{d i r}\left(\frac{\cos (i)}{\cos (s z)}+V_{t} \rho_{a d j}\right)+E_{d i f}\left(k * \frac{\cos (i)}{\cos (s z)}+(1-k) V_{d}+V_{t} \rho_{a d j}\right)
$$

For a specific bands b, the surface reflectance $\rho$ can be calculated by the following formula:

$$
\rho=\frac{\left(L-L_{p}\right) \pi}{E_{\text {total }}^{i} \tau_{1}}
$$

$L_{p}$ is the path irradiance, $\tau_{1}$ is the total transmission of atmosphere, $L$ is the irradiance at the satellite.
So the physical model to correct the reflectance of mountain area is:

$$
\rho=\frac{\left(a * D N+b-L_{p}\right) \pi}{\left(E_{d i r}\left(\frac{\cos (i)}{\cos (s z)}+V_{t} \rho_{a d j}\right)+E_{d i f}\left(k \frac{\cos (i)}{\cos (s z)}+(1-k) V_{d}+V_{t} \rho_{a d j}\right)\right) \tau_{1}}
$$

$a$ and $b$ is the calibrate parameters of satellite image. The $6 \mathrm{~S}$ model enables to simulate the signal observed by a satellite sensor for a lamebrain target at sea level altitude(Vermote, Tanre, Deuze, Herman, \& Morcrette, 1997).We can get $E_{\text {dir }} E_{d i f}, L_{p}$ from $6 \mathrm{~S}$ model in a given atmosphere condition. For an image with accurate calibrate parameters we can get the corrected reflectance of mountain surface, such as Landsat8 OLI image. In order to adapt to situations where accurate calibration and atmosphere parameters are not available. Formula (6) can be transformed to the following form:

$$
\begin{aligned}
& \tau_{1} \rho E_{d i r}\left(\frac{\cos (i)}{\cos (s z)}+V_{t} \rho_{a d j}\right) /\left(b-L_{p}\right)+ \\
& \tau_{1} \rho E_{d i f}\left(k \frac{\cos (i)}{\cos (s z)}+(1-k) V_{d}+V_{t} \rho_{a d j}\right) /\left(b-L_{p}\right)- \\
& (a * D N) \pi /\left(b-L_{p}\right)=\pi
\end{aligned}
$$

Let

The formula is :

$$
\begin{gathered}
C_{1}=\rho\left(\frac{\cos (i)}{\cos (s z)}+V_{t} \rho_{a d j}\right) \quad C_{2}=\rho\left(k \frac{\cos (i)}{\cos (s z)}+(1-k) V_{d}+V_{t} \rho_{a d j} \quad C_{3}=1\right. \\
X_{1}=\frac{\tau_{1} E_{d i r}}{\mathrm{a} \pi} \quad X_{2}=\frac{\tau_{1} E_{d i f}}{\mathrm{a} \pi} \quad X_{3}=\frac{L_{p}-b}{a} \\
C_{1} X_{1}+C_{2} X_{2}+C_{3} X_{3}=D N \\
\rho=\frac{D N-X_{3}}{X_{1}\left(\frac{\cos (i)}{\cos (s z)}+V_{t} \rho_{a d j}\right)+X_{2}\left(k \frac{\cos (i)}{\cos (s z)}+(1-k) V_{d}+V_{t} \rho_{a d j}\right)}
\end{gathered}
$$

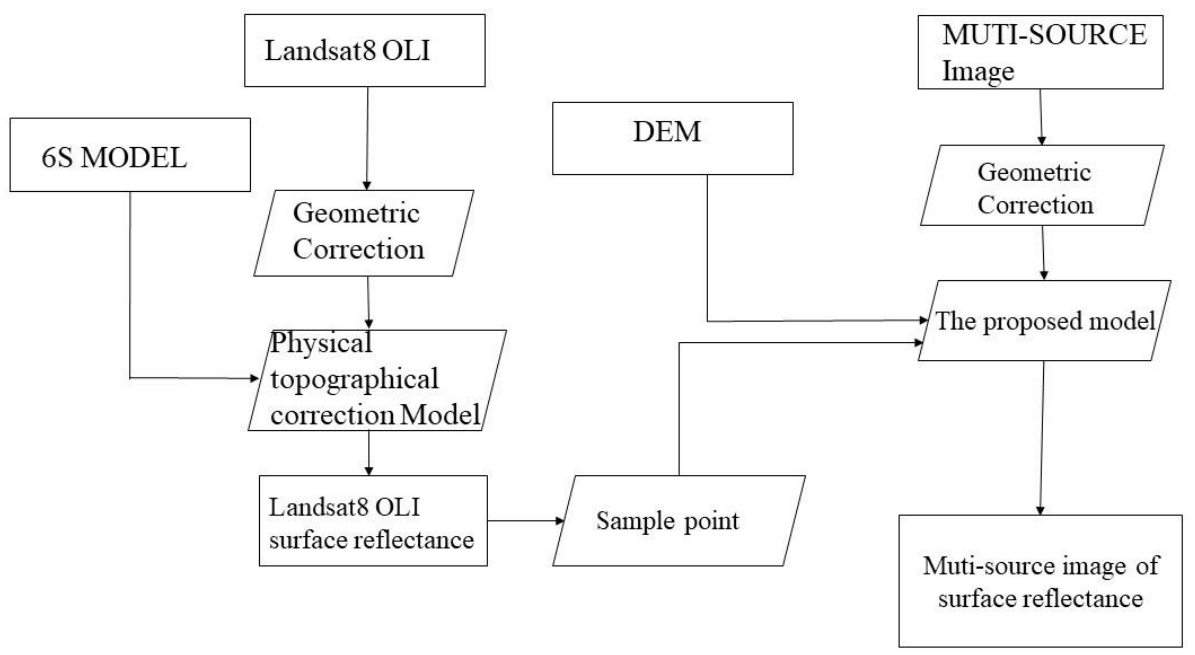

Fig.2.1 flow chart of this model

Among them $C_{\mathrm{i}}(i=1,2,3)$ is the parameters determined by surface reflectance and surface geometric conditions, $X_{\mathrm{i}} \quad(i=1,2,3)$ is determined by sensors and atmosphere conditions. Figure 2.1 shows the flow chart of this model. 
In this study, we choose Landsat8 as base image with accurate calibrate parameters, with the DEM of this region and physical model in (6), we can get topographic corrected surface reflectance. In the study region, we choose some sample point, and construct linear equations. By solving the least squares solution to this linear system of equations then get

\subsection{Data of study area}

Our study area is Saihanba state forest farm of Chengde City, Hebei Province, China.
$X_{\mathrm{i}}(i=1,2,3)$,bring these parameters into equation 6 for multisource image, we can get topographic corrected surface reflectance for multi-source image without accurate calibrate parameters.

The study area is located in the transitional zone of the North China Plain to the Mongolian Plateau, with a great surface fluctuation. The altitude ranges from $1010 \mathrm{~m}$ to $1939.9 \mathrm{~m}$, the average slope is $7.461^{\circ}$, the maximum slope is $56.028^{\circ}$.
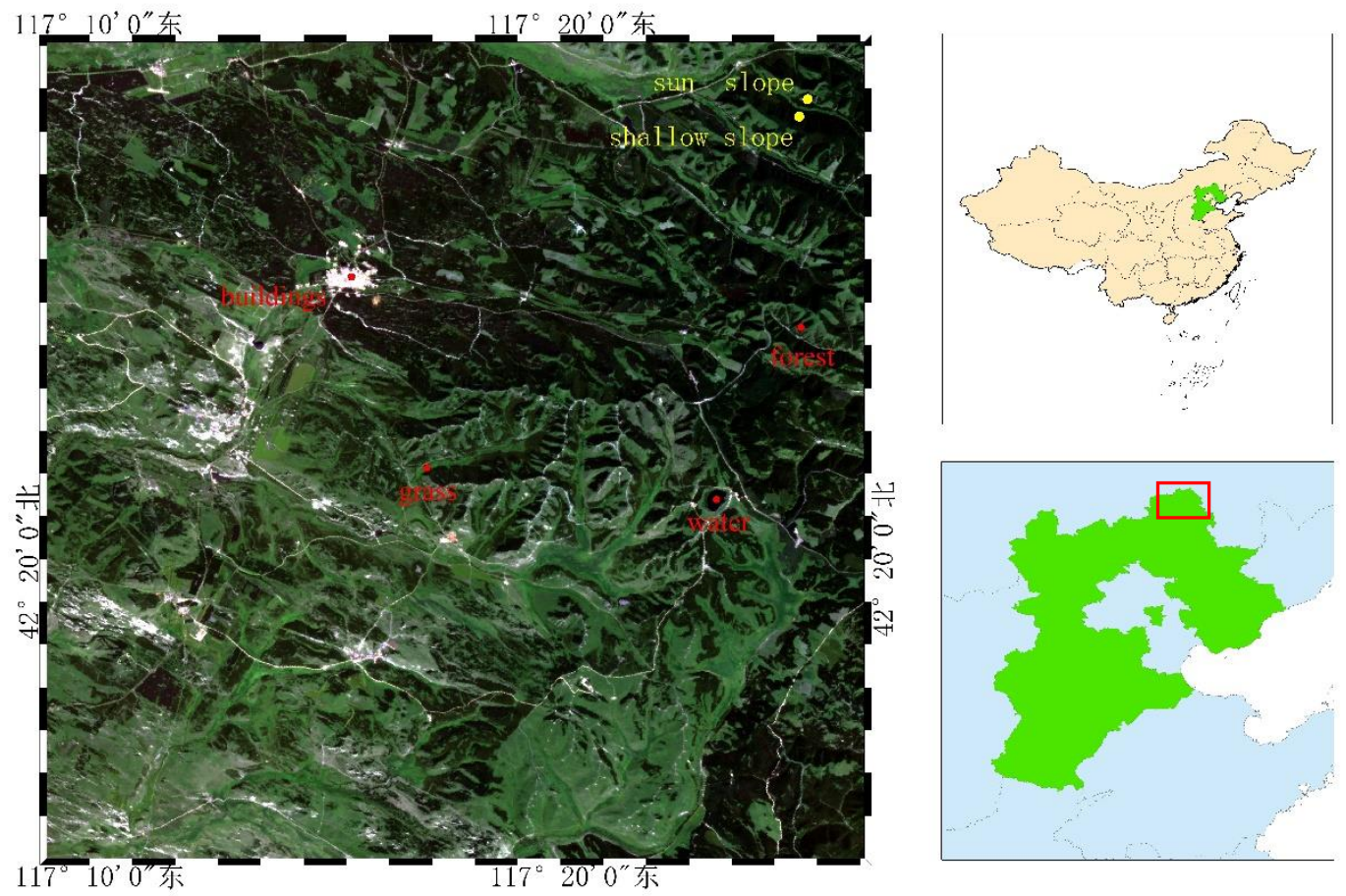

Fig.2.2 . the study area

The other important data been used is DEM. We choose SRTM with $30 \mathrm{~m}$ resolution

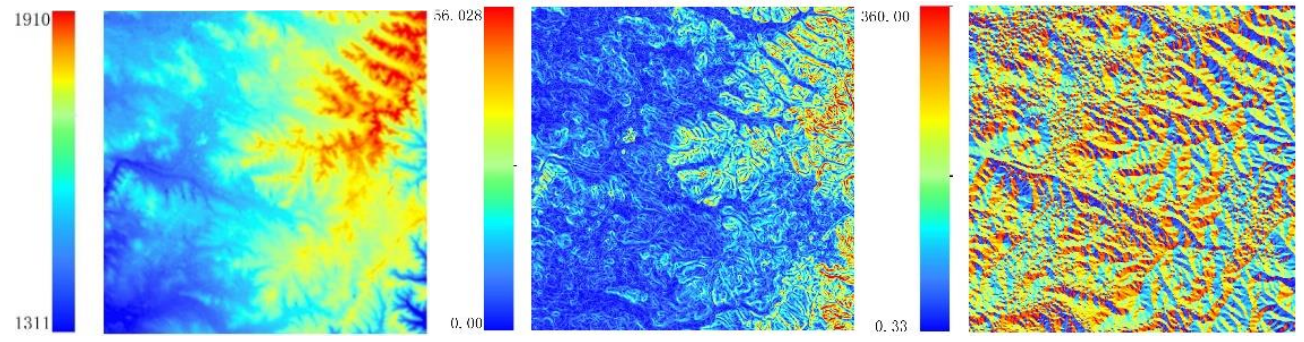

Fig.2.3. the elevation (a), slope (b) and aspect(c) of study region

The base data we used is acquired by Landsat8 OLI in 2016-0808. The multi-source data we used is GF and HJ. The basic parameters of these satellites are shown in the table2.1.

The GF1 and HJ1 are two multispectral satellite of China. The GF1 was launched in 2013, and the HJ1 was launched in 2008. They have wide view angle and high time resolution. But because they have worked for a long time, so their calibration is less accurate. 
ISPRS Annals of the Photogrammetry, Remote Sensing and Spatial Information Sciences, Volume IV-3, 2018 ISPRS TC III Mid-term Symposium “Developments, Technologies and Applications in Remote Sensing”, 7-10 May, Beijing, China

Table2.1. Comparison of basic parameters between Landsat8-OLI data and GF1-WFV4 and HJ1B-CCD2 data

\begin{tabular}{llll}
\hline & Landsat-OLI & GF1-WFV4 & HJ1-CCD1 \\
\hline Sun azimuth(degree) & 138.785 & 169.928 & 315 \\
Sun zenith(degree) & 31.39 & 25.735 & 32 \\
Resolution(m) & 30.00 & 17.42 & 30.00 \\
Time & $2016-08-08-10: 53: 09.02$ & $2016-08-07-12: 02: 15$ & $2016-08-08$ \\
\hline
\end{tabular}

\section{RESULT ANALYSIS}

\subsection{Vissual Analysis}

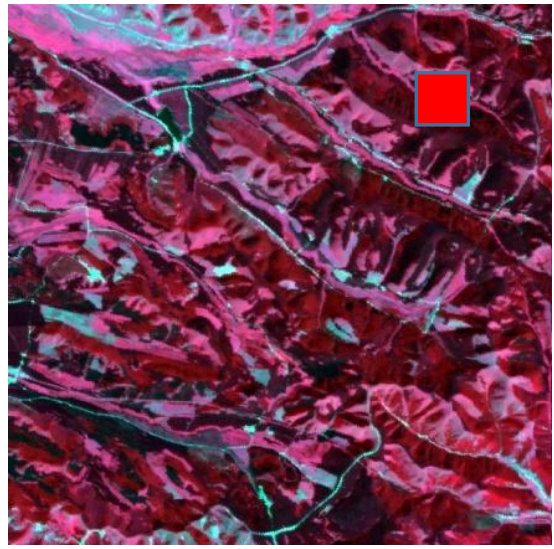

(a)

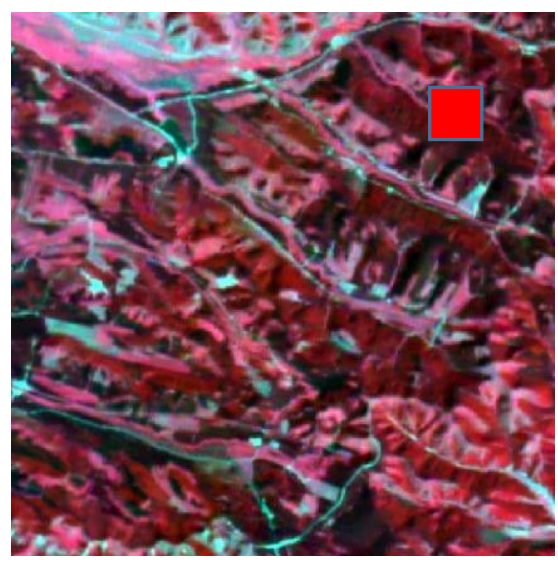

(c)

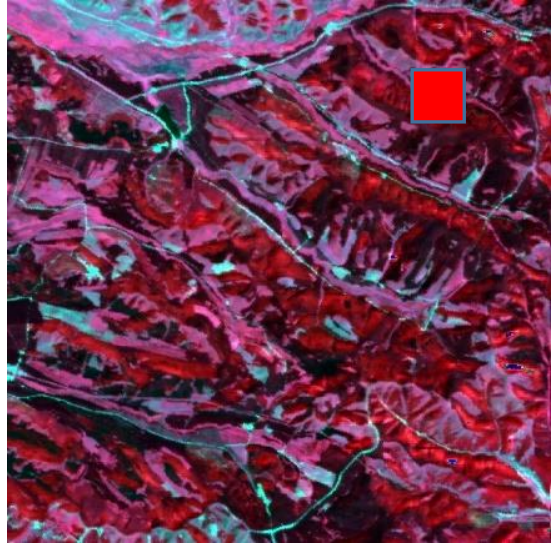

(b)

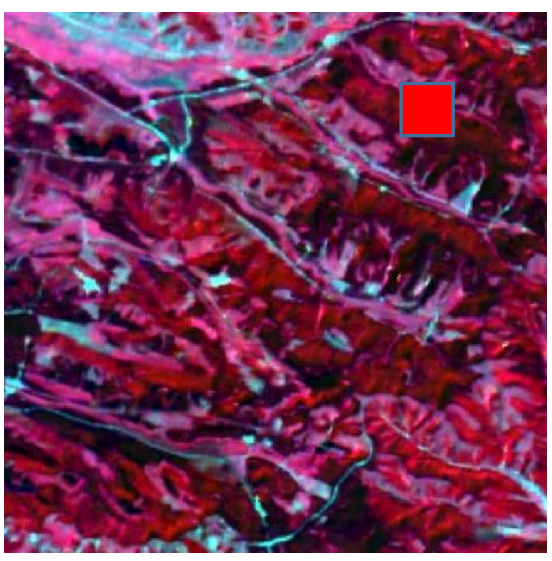

(d)

Fig.3.1. These pictures shows the DN value before topographic correction of GF(a) and HJ(c) and the surface reflectance after topographic correction of $\mathrm{GF}(\mathrm{b})$ and $\mathrm{HJ}(\mathrm{d}), 4,3,2$ false composite.

The result shows that after topographic correction, the brightness of slope face away from the sun has increased, and the brightness

\subsection{Reflectance Analysis}

The figure 3.2 shows the reflectance of four land cover type after topographic correction with $\mathrm{HJ}(\mathrm{a})$ and $\mathrm{GF}(\mathrm{b})$. The position of the point has marked in figure 2.2 with red point. The shape of the curve is correct, the reflectance of water has a low value of slope face from the sun nearly has no change. the difference between sunny slope and shady slope has reduced. For GF data there are some overcorrections when the slope is too large, but for $\mathrm{HJ}$ there are no overcorrections.

and building has a high value, the reflectance of forest and grass has a high value in band 4 and a low value in band1, band 2 and band3. The difference is the reflectance of building with $\mathrm{HJ}$ in band 3 is higher than in band 4 but in contrast with GF. 


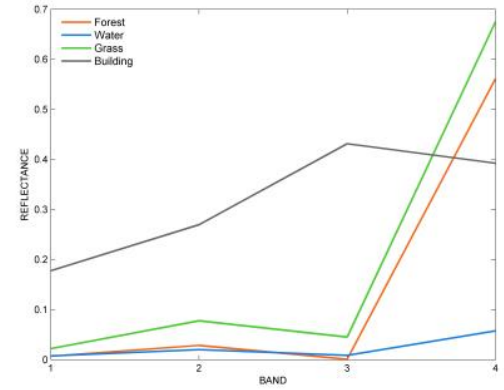

(a)

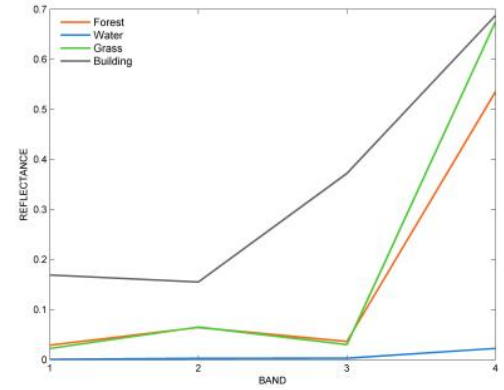

(b)

Fig.3.2the reflectance of four land cover type after topographic correction with $\mathrm{HJ}(\mathrm{a})$ and $\mathrm{GF}(\mathrm{b})$

The figure 3.3 shows the curve of reflectance with no topographic correction and topographic correction with this model. As is shown in the figure (2.2), the sun slope and the shallow slope has been marked with yellow point. The slope of the shallow slope and the sun slope is 18.64 degree and 19.54 degree, and the aspect of the shallow slope and the sun slope is 14degree and 209degree. Figure (a) shows that there is a large difference in reflectance between sunny and shady slopes without considering the impact of the terrain. After the GF and HJ are corrected by the model in this paper, the reflectance difference becomes smaller From the curve, the reflectance have changed in shadow slope and sunny

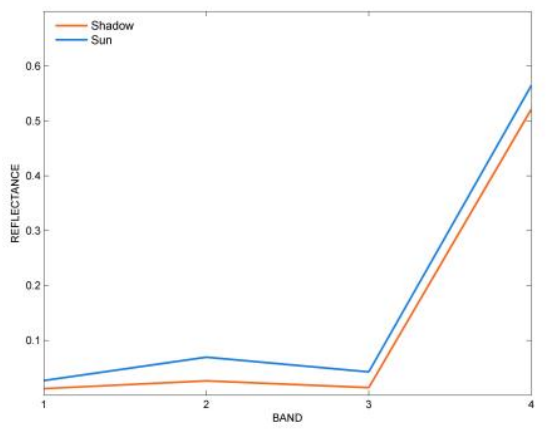

(a) slope, but the reflectance in shadow changed a lot. In general, the difference in reflectance between the shady slope and the sunny slope becomes smaller.

Covariance represents the correlation between the two datasets, if the two datasets are completely correlated, then their covariance is 1 , if they are completely not correlated, their covariance is 0 . The larger the covariance, the greater the correlation between the two datasets. The tables below shows the covariance between the DN value of GF and $\mathrm{HJ}$ and the reflectance of Landsat8OLI image in band 4 and the covariance between the reflectance of $\mathrm{HJ}$ and the reflectance of Landsat8OLI image in band 4 .

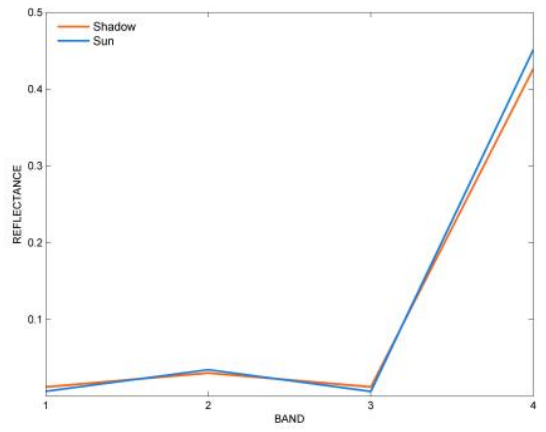

(b)

Fig.3.3. the reflectance of shadow slope and Sun slope with no topographic correction and topographic with this model, GF (a), HJ (b)

Table.3.1 The covariance between multi-source image and Landsat8 OLI image.

\begin{tabular}{ccc}
\hline & HJ & GF \\
\hline Before topographic corrected & 0.670 & 0.782 \\
\hline After topographic corrected & 0.806 & 0.904 \\
\hline
\end{tabular}

The table shows the covariance between multi-source image and Landsat 8 OLI image in band4,the covariance of $\mathrm{HJ}$ has increased from 0.67 to 0.8 , the covariance of GF has increased from 0.78 to 0.90.this resents that after topographic correction ,the covariance between HJ,GF and Landsat8OLI image becomes larger, because the reflectance of Landsat8OLI if the result of physical model, the reflectance of HJ and GF is the result of this semi-empirical model, so the higher the covariance is, the better the result of semi-empirical is.

Table.3.2. The topographical correlation factor between multi-source image and Landsat8 OLI image.

\begin{tabular}{llllll}
\hline & & Band 1 & Band2 & Band3 & Band4 \\
\hline GF & No correction & 0.014 & 0.024 & 0.0194 & 0.0751 \\
& Correction & 0.012 & 0.0102 & 0.0126 & 0.0128 \\
HJ & No correction & 0.0136 & 0.0297 & 0.0226 & 0.095 \\
& Correction & 0.0136 & 0.0135 & 0.0134 & 0.0134 \\
\hline
\end{tabular}


The topographical correlation factor is obtained by calculating the slope of the linear regression equation of the surface reflectance relative to the cosine of the earth's surface relative to the angle of incidence. It indicates the extent to which the surface reflectance is affected by the terrain. Ideally, the surface reflectance is completely unaffected by the terrain. In this case, the slope of the linear regression equation is 0 , and the smaller slope indicates that the surface reflectance is less influenced by

\subsection{Evaluate Classification Accuracy}

On the base of topographic correction, and we classify the surface coverage of the study area, the type of surface can be divided into 4 categories: forest, grassland, building and bare soil. The table the terrain, which in turn indicates that the reflectance is more affected by the terrain.

The table.3.2 shows the topographical correlation factor of GF and $\mathrm{HJ}$ in forest area. As can be seen from the table, for GF, the factor is 0.0751 before correction, and it decreased to 0.0128 after correction. In all four bands ,the result of band 4 is the best, while band1 is strongly affected by atmosphere condition, the result is not very good.

Table.3.3. Overall accuracy and kappa before and after topographic correction

\begin{tabular}{lllll}
\hline & Before correction & After correction \\
\hline data & GF & HJ & GF & HJ \\
Overall Accuracy & $93.4496 \%$ & $76.5095 \%$ & $93.7144 \%$ & $90.8201 \%$ \\
Kappa & 0.8592 & 0.6562 & 0.8678 & 0.8607 \\
\hline
\end{tabular}

As can be seen from the table.3.3, in general after topographic correction, for GF the improvement of kappa and overall accuracy is not so obvious. But for $\mathrm{HJ}$, there is a certain

\section{CONCLUSION AND DISCUSSION}

\subsection{Conclusion}

The premise of quantitative application of remote sensing in the mountains is accurate atmospheric correction and topographic correction. But for some data source, we cannot get the accurate calibration parameters. Using the semi-empirical terrain correction model developed in this paper and the reference image, the surface reflectance after atmospheric topography correction can be obtained directly from the DN value of the original image. Using domestic GF and HJ to verify the model, draw the following conclusions in this paper:

(1): By calculating the covariance between physical based Landsat8 OLI image, the result shows that the model in this paper can effectively reduce the terrain effect for GF and HJ.

(2): By calculating the Terrain correlation factor, this model can effectively reduce the terrain correlation factor, and the band 4 shows the best result in this model.

\section{ACKNOWLEDGEMENTS}

This paper is supported by "the Fundamental Research Funds for the Central Universities

\section{REFERENCE}

Chen, Y., Hall, A., \& Liou, K. N. (2006). Application of threedimensional solar radiative transfer to mountains. Journa $1 \mathrm{Of}$ Geophysica Research-Atmospheres, https://doi.org/10.1029/2006JD007163

Gu, D., \& Gillespie, A. (1998). Topographic normalization of landsat TM images of forest based on subpixel Sun-canopysensor geometry. Remote Sensing Of Environment https://doi.org/10.1016/S0034-4257(97)00177-6

Sandmeier, S., \& Itten, K. I. (1997). A physically-based model to shows the overall accuracy and kappa before and after topographic correction. improvement in overall accuracy and Kappa. The overall accuracy has increased from $76.5 \%$ to $90.82 \%$, and the kappa has increased from 0.65 to 0.86 ,this means the classification result for $\mathrm{HJ}$ is better than GF.

(3): After topographic correction, the classification accuracy has increased in some extent. For HJ, the model can improve the classification accuracy to a great extent, but for GF, the improvement is not so obvious.

\subsection{Discussion}

Although the research shows that this model can effectively reduce the influence of topography on the surface reflectance, there are still some problems, mainly in the following aspects:

(1): There is no ground-based surface reflectance and land cover classification map to validate the model, so the accuracy of the model is not fully assessed.

(2): Because the sample point wo choose is randomly generated, so the reflectance of the same point in two different image may not be the same, these may cause the change of the result.

(3): Due to the spectrum conversion function of Landsat and HJ and GF are not correctly the same, so ,in the same band, the reflectance of GF, HJ, and Landsat are not correctly the same

correct atmospheric and illumination effects in optical satellite data of rugged terrain. Ieee Transactions on Geoscience And Remote Sensing, https://doi.org/10.1109/36.581991

Soenen, S. A., Peddle, D. R., \& Coburn, C. A. (2005). SCS+C: A modified sun-canopy-sensor topographic correction in forested terrain. Ieee Transactions on Geoscience And Remote Sensing, https://doi.org/10.1109/Tgrs.2005.852480

Vermote, E. F., Tanre, D., Deuze, J. L., Herman, M., \& Morcrette, J. J. (1997). Second Simulation of the Satellite Signal in the Solar Spectrum, 6S: An overview. Ieee Transactions on Geoscience And Remote Sensing, https://doi.org/10.1109/36.581987

Yu K, Liu S, Zhao Y. CPBAC: A quick atmospheric correction method using the topographic information. Remote Sensing of 
Environment, https://doi.org/10.1016/j.rse.2016.08.010

Li F, Jupp D L B, Thankappan M, et al. A physics-based atmospheric and BRDF correction for Landsat data over mountainous terrain. Remote Sensing of Environment, https://doi.org/10.1016/j.rse.2012.06.018

Sola I, González-Audícana M, Álvarez-Mozos J. Multicriteria evaluation of topographic correction methods. Remote Sensing of Environment, https://doi.org/10.1016/j.rse.2016.07.002

Young N E, Anderson R S, Chignell S M, et al. A survival guide to Landsat preprocessing. Ecology, 2017, 98(4):920-932. https://doi.org/10.1002/ecy.1730

Chander G, Markham B L, Helder D L. Summary of current radiometric calibration coefficients for Landsat MSS, TM, ETM+, and EO-1 ALI sensors[J]. Remote Sensing of Environment, https://doi.org/10.1016/j.rse.2009.01.007

Proy C, Tanre D, Deschamps P Y. Evaluation of topographic effects in remotely sensed data.[J]. Remote Sensing of Environment, https://doi.org/10.1016/0034-4257(89)90044-8 